\title{
Danicopan: an oral complement factor D inhibitor for paroxysmal nocturnal hemoglobinuria
}

Antonio M. Risitano, ${ }^{1,2}$ Austin G. Kulasekararaj, ${ }^{3,4}$ Jong Wook Lee, ${ }^{5}$
Jaroslaw P. Maciejewski, ${ }^{6}$ Rosario Notaro,, 8 Robert Brodsky, ${ }^{9}$
Mingjun Huang, ${ }^{10}$ Michael Geffner $^{11}$ and Peter Browett ${ }^{12}$

${ }^{1}$ Federico II University of Naples, Naples, Italy; ${ }^{2}$ AORN Moscati, Avellino, Italy; ${ }^{3}$ King's College Hospital-NHS Foundation Trust, NIHR/Wellcome King's Clinical Research Facility, London, UK; ${ }^{4}$ King's College London, London, UK; ${ }^{5}$ Seoul St. Mary's Hospital, The Catholic University of Korea, Seoul, Republic of Korea; ${ }^{6}$ Cleveland Clinic, Cleveland, $\mathrm{OH}$, USA; ${ }^{7}$ Core Research Laboratory, Istituto per lo Studio, la Prevenzione e la Rete Oncologica (ISPRO), Firenze, Italy; ${ }^{8}$ Azienda Ospedaliera-Universitaria Careggi, Firenze, Italy; ${ }^{9}$ Johns Hopkins University School of Medicine, Baltimore, MD, USA; ${ }^{10}$ Achillion, Inc., A Subsidiary of Alexion Pharmaceuticals, Inc., New Haven, CT, USA and Alexion Pharmaceuticals, New Haven CT, USA; ${ }^{11}$ Achillion Inc., A Subsidiary of Pharmaceuticals, Inc., Blue Bell, PA, USA and ${ }^{12}$ University of Auckland, Auckland, New Zealand

Presented as an oral presentation at the $24^{\text {th }}$ European Hematology Association Congress, Amsterdam, NL, June 15, 2019. Presented as a poster presentation at the $61^{\text {st }}$ annual meeting of the American Society of Hematology, Orlando, FL, USA December 8, 2019.

\section{Correspondence:}

ANTONIO M. RISITANO

amrisita@unina.it

Received: June 3, 2020.

Accepted: October 6, 2020.

Pre-published: October 29, 2020.

https://doi.org/10.3324/haematol.2020.261826

(C)2021 Ferrata Storti Foundation

Material published in Haematologica is covered by copyright. All rights are reserved to the Ferrata Storti Foundation. Use of published material is allowed under the following terms and conditions:

https://creativecommons.org/licenses/by-nc/4.0/legalcode. Copies of published material are allowed for personal or internal use. Sharing published material for non-commercial purposes is subject to the following conditions:

https://creativecommons.org/licenses/by-nc/4.0/legalcode, sect. 3. Reproducing and sharing published material for commercial purposes is not allowed without permission in writing from the publisher.

\section{ABSTRACT}

$\mathcal{D}$ aroxysmal nocturnal hemoglobinuria $(\mathrm{PNH})$ is characterized by complement-mediated intravascular hemolysis due to the absence of complement regulators CD55 and CD59 on affected erythrocytes. Danicopan is a first-in-class oral proximal, complement alternative pathway factor $\mathrm{D}$ inhibitor. Therapeutic factor $\mathrm{D}$ inhibition was designed to control intravascular hemolysis and prevent C3-mediated extravascular hemolysis. In this open-label, phase II, dose-finding trial, ten untreated $\mathrm{PNH}$ patients with hemolysis received danicopan monotherapy (100-200 mg thrice daily). Endpoints included changes in the concentrations of lactate dehydrogenase (LDH) at day 28 (primary endpoint), of LDH at day 84, and of hemoglobin. Safety, pharmacokinetics/pharmacodynamics, and patient-reported outcomes were assessed. Ten patients reached the primary endpoint; two later discontinued treatment: one because of a serious adverse event (elevated aspartate aminotransferase/alanine aminotransferase coincident with breakthrough hemolysis, resolving without sequelae) and one for personal reasons unrelated to safety. Eight patients completed treatment. Intravascular hemolysis was inhibited, as demonstrated by a mean decrease of $\mathrm{LDH}$ (5.7 times upper limit of normal [ULN] at baseline vs. 1.8 times ULN at day 28 and 2.2 times ULN at day 84 ; both $P<0.001)$. Mean baseline hemoglobin, $9.8 \mathrm{~g} / \mathrm{dL}$, increased by 1.1 (day 28) and 1.7 (day 84) g/dL (both $P<0.005$ ). No significant C3 fragment deposition occurred on glycosylphosphatidylinositol-deficient erythrocytes. Mean baseline Functional Assessment of Chronic Illness Therapy-Fatigue score, 34, increased by 9 (day 28) and 13 (day 84) points. The most common adverse events were headache and upper respiratory tract infection. These phase II, monotherapy data show that proximal inhibition with danicopan provides clinically meaningful inhibition of intravascular hemolysis and increases hemoglobin concentration in untreated $\mathrm{PNH}$ patients, without evidence of C3-mediated extravascular hemolysis. This trial was registered at www.clinicaltrials.gov (\#NCT03053102). 


\section{Introduction}

Paroxysmal nocturnal hemoglobinuria $(\mathrm{PNH})$ is a rare hematologic disease characterized by chronic intravascular hemolysis, severe thrombophilia, and bone marrow failure. ${ }^{1} \mathrm{PNH}$ is due to somatic mutations of the phosphatidylinositol $\mathrm{N}$-acetylglucosaminyltransferase subunit A (PIGA) gene in hematopoietic stem cells that impair biosynthesis of glycosylphosphatidylinositol (GPI) anchors and surface expression of GPI-linked proteins. ${ }^{2-4}$ While bone marrow failure is secondary to T-cell-mediated immune attack that spares PIGA-mutated hematopoietic stem cells, ${ }^{5,6}$ both hemolysis and thromboembolism are complement-mediated. GPI-deficient erythrocytes (and platelets) lack GPI-linked complement regulators $\mathrm{CD}_{5} 5^{7}$ and $\mathrm{CD} 59^{8}$ and are exquisitely vulnerable to complement activation, which occurs continuously and spontaneously due to "C3 tick-over" and acutely with specific triggers. PNH treatment was revolutionized by introduction of the terminal complement C5 inhibitor eculizumab, which has proven effective in addressing intravascular hemolysis ${ }^{10,11}$ and thromboembolism, ${ }^{12}$ with a significant impact on long-term survival. ${ }^{13,14}$ Recently, a new long-acting C5 inhibitor dosed every 8 weeks, ravulizumab, has demonstrated non-inferiority to eculizumab in controlling intravascular hemolysis. ${ }^{15,16}$ Although the significant benefits of $\mathrm{C} 5$ inhibition in the treatment of PNH patients have been clearly demonstrated, the hematologic benefit may be hampered by the emergence of C3-mediated extravascular hemolysis from early phases of complement activation, ${ }^{17,18}$ which C5 inhibition cannot address. ${ }^{19-21}$ Thus, alternative strategies of complement inhibition are required to improve $\mathrm{PNH}$ treatment, and agents are in development to address this and other unmet patients' needs, including improved convenience. ${ }^{22}$

The complement cascade has three activating pathways (alternative, classical, and lectin-mannose) that merge at the key complement component C3; from this level (which is amplified by alternative pathway [AP] proteins), the effector pathway starts, with generation of anaphylatoxins and the membrane attack complex (MAC). ${ }^{23}$ Novel strategies of therapeutic complement inhibition in development aim to intercept the complement cascade upstream of $\mathrm{C} 5$, some targeting upstream at pathway-specific initiating events. ${ }^{22}$ During AP initiation, the serine protease complement factor $\mathrm{D}$ cleaves factor $\mathrm{B}$, leading to AP C3 convertase generation. Danicopan (ACH-4471, ACH-044471, ALXN2040) is a first-in-class, oral, smallmolecule factor $\mathrm{D}$ inhibitor that prevents new AP C3 convertase formation..$^{24}$ Consequently, proximal inhibition at the level of factor D blocks AP-initiated upstream events and up to $80 \%$ of classical or lectin pathway-initiated downstream events via amplification-loop inhibition. ${ }^{25}$ In vitro, danicopan inhibited both AP-mediated hemolysis and C3 fragment deposition on red blood cells from PNH patients. ${ }^{2}$ Phase I data from healthy human volunteers in single and multiple ascending-dose trials showed that danicopan was generally well tolerated and could achieve inhibition of AP complement activity. ${ }^{26}$ This work identified danicopan $200 \mathrm{mg}$ thrice daily (tid) as a safe and effective dose/regimen. ${ }^{26}$ For $\mathrm{PNH}$, targeting factor $\mathrm{D}$ inhibition with a small molecule represents a potentially important treatment advancement because proximal AP inhibition may disable both terminal complement activation (inhibiting MAC-mediated intravascular hemolysis) and C3 fragment opsonization (preventing extravascular hemolysis), with additional convenience of oral administration.

We investigated the factor $\mathrm{D}$ inhibitor danicopan as single-agent treatment for untreated $\mathrm{PNH}$, aiming to control intravascular hemolysis while preventing C3-mediated extravascular hemolysis.

\section{Methods}

\section{Study design}

This international, open-label, single-arm, dose-finding, phase II study investigated danicopan in patients with hemolytic PNH not receiving complement inhibitor treatment. This trial was approved by regulatory agencies/local ethics committees and conducted according to International Conference on Harmonisation and Good Clinical Practice Standards. Achillion, Inc., a subsidiary of Alexion Pharmaceuticals, Inc., designed and sponsored the study, with advice from the investigators. All participants provided written informed consent. The trial is registered at $w$ ww.clinicaltrials.gov as \#NCT03053102.

\section{Patients}

This study was conducted from March 2017 to November 2018 and involved adults with untreated $\mathrm{PNH}$. To be enrolled, patients had to have hemoglobin $<12 \mathrm{~g} / \mathrm{dL}$ (and adequate reticulocytosis according to the investigator), GPI-deficient granulocytes or type III erythrocyte clone size $\geq 10 \%$, lactate dehydrogenase (LDH) $\geq 1.5$ times upper limit of normal (ULN), platelet counts $\geq 50 \times 10^{9} / \mathrm{L}$, and willingness to be vaccinated for $N$. meningitidis, $H$. influenzae, and $S$. pneumoniae. Investigators used their clinical judgement to assess whether patients had enough bone marrow capacity to derive potential benefit by comparing the level of pre-entry hemoglobin to the absolute reticulocyte count. None of the subjects was receiving eculizumab, because of lack of availability and/or the patients' willingness.

\section{Treatment}

Patients received oral danicopan at a starting dose of $100 \mathrm{mg}$ or $150 \mathrm{mg}$ tid. The starting dose was based on phase I data showing that danicopan doses of 200 to $600 \mathrm{mg}$ reached peak plasma levels within 1 to $2 \mathrm{~h}$ and were well-tolerated, and that $200 \mathrm{mg}$ tid was effective on $\mathrm{PNH}$ red blood cells. ${ }^{22,26}$ Dose escalations were permitted based on hemolysis control, assessed by LDH, per investigator assessment in stepwise increments up to $200 \mathrm{mg}$ tid. Dose escalation criteria for the first 28 days were specified in the study protocol with potential dose escalation points occurring at days 7 and 14 (Online Supplementary Appendix). Dose escalation was permitted thereafter and was done in consultation between the investigator and sponsor based on the hemoglobin response; absolute reticulocyte count, $\mathrm{LDH}$, and indirect bilirubin were reviewed to evaluate evidence for possible additional clinical benefit from dose escalation. Because this study was the first treatment experience with danicopan in $\mathrm{PNH}$ patients, dose escalations were approached cautiously, especially when moving to the higher doses in the study (from 150 to $175 \mathrm{mg}$ and 175 to $200 \mathrm{mg}$ ) or if alanine aminotransferase levels had fluctuated relative to the baseline value. Patients were instructed to take their medication approximately every $8 \mathrm{~h}$. On study center days, when blood for laboratory tests was drawn, the morning dose was to be administered in the study center by the site personnel following safety and pharmacokinetic assessments. Additionally, patients were instructed to take each dose with food, including prior to intensive sampling for pharmacokinetic studies on days 1,13 , and 28 . The planned duration of treatment was 84 days; patients completing 
treatment with clinical benefit entered a long-term extension study (ClinicalTrials.gov; NCT03181633).

\section{Endpoints}

The primary efficacy endpoint was change in LDH concentration from baseline at day 28 . Secondary efficacy parameters were change of hemoglobin concentration from baseline at days 28 and 84 and LDH change from baseline at day 84 . Safety, tolerability, pharmacokinetics, and pharmacodynamics were also investigated. Laboratory assessments comprised hematology, chemistry and urinalysis. Patient-reported outcomes were measured at baseline and during the study via the validated Functional Assessment of Chronic Illness Therapy (FACIT)-Fatigue scale. Haptoglobin, bilirubin, reticulocytes, GPI-deficient clone size of erythrocytes (type III) and granulocytes (type II and III unless type III values provided), and percentage of C3 fragment-coated erythrocytes were also monitored. Soluble C5b-9 was evaluated as an exploratory endpoint (non-GLP); C5a was not monitored. Transfusion history up to 3 years prior to and during the study was collected.

\section{Assay methods}

Plasma danicopan concentrations were determined by liquid chromatography. Pharmacodynamics were determined by measuring serum AP activity with an AP Wieslab assay. Plasma Bb concentration, serum factor $\mathrm{D}, \mathrm{C} 3$, and $\mathrm{C} 4$ concentrations were also monitored. All aforementioned complement tests were conducted in a central laboratory using commercial kits. C3 fragment deposition on erythrocytes was measured using flow cytometry with FITC-conjugated anti-human C3d antibody (see the Online Supplement for details).

\section{Statistical analysis}

This was a proof-of-concept, first-in-patients, exploratory, phase II study. The sample size was determined based on the very limited number of untreated $\mathrm{PNH}$ patients and the exploratory nature of this study to evaluate effectiveness of danicopan. Given the small sample size, only descriptive and exploratory statistics were utilized to present results for continuous biochemical and quality-of-life measurements. Patients who discontinued treatment during the trial were not replaced. Missing values were not imputed. To summarize categorical data, frequency counts and percentages are presented. The Pearson correlation coefficient (Pearson r) was used to examine the relationship between two variables. The quantitative analysis between pharmacokinetics (plasma danicopan concentration) and pharmacodynamics (AP inhibition) was conducted with nonlinear regression using the simple $\mathrm{E}_{\max }$ dose-response equation (Prism 5.02, GraphPad Software, La Jolla, CA, USA).

\section{Results}

\section{Patients' characteristics}

Eleven patients were screened. Ten untreated patients with hemolytic PNH were enrolled and received danicopan. These patients' baseline characteristics are presented in Table 1 and Online Supplementary Table S1. Their median age was 33 years (range, 17-63 years) and median disease duration was 5.9 years (range, $0-14$ years). The mean GPI-deficient clone size was $79 \%$ for granulocytes and $32 \%$ for erythrocytes. Overt hemolysis was demonstrated by elevated $\mathrm{LDH}$ levels $(1416 \pm 540 \mathrm{U} / \mathrm{L}$, corresponding to $5.7 \pm 2.17$ times ULN), increased reticulocyte count $(154 \pm 69 \times 109 / \mathrm{L})$, increased total bilirubin $(1.3 \pm 0.74$ $\mathrm{mg} / \mathrm{dL})$, and reduced haptoglobin $(5.8 \pm 2.9 \mathrm{mg} / \mathrm{dL})$. The baseline hemoglobin concentration was heterogeneous among patients $(9.8 \pm 1.8 \mathrm{~g} / \mathrm{dL})$; two patients received transfusions in the 12 weeks preceding study entry, with one of these patients also having a medical history of aplastic anemia.

\section{Study disposition and safety}

The disposition of patients in the study is shown in Online Supplementary Figure S1. Two patients started danicopan at $100 \mathrm{mg}$ tid and increased to $150 \mathrm{mg}$ tid, and eight started at $150 \mathrm{mg}$ tid. Increases to $175 \mathrm{mg}$ and 200 mg tid were performed in eight and four patients, respectively. All ten patients reached day 28 and are included in the primary endpoint evaluation. Two discontinued before day 84: one because of a serious adverse event, elevated aspartate aminotransferase/alanine aminotransferase coincident with breakthrough hemolysis, which resolved without sequelae; the other withdrew for personal reasons unrelated to safety. All patients were evaluated until they left the study or reached day $84(n=8)$. Nine patients (90\%) developed at least one adverse event during treatment; only one was serious (described above). In total, 38 unique treatment-emergent adverse events were recorded, of which four were considered possibly related and two probably related to danicopan. The most frequent events were PNH-related (hemolysis and its signs or symptoms) and infections, usually of the upper respiratory tract (Table 2). With few exceptions, adverse events were mild and resolved during the study. There were no clinically significant changes in other key laboratory parameters during treatment (Online Supplementary Table S2).

\section{Pharmacokinetics and pharmacodynamics}

Danicopan was bioavailable with dose-proportional exposure (peak serum concentration $\left[\mathrm{C}_{\max }\right]$ and area under the curve [AUC]) at 100, 150, and $175 \mathrm{mg}$ tid doses, demonstrated by intensive pharmacokinetic and pharmacodynamic profiling on days 6, 13, and 20 (Figure 1A; Online Supplementary Table S3), whereas trough concentrations assessed biweekly at single time points from days 28 to 84 were variable (Online Supplementary Table S4). Trough drug concentration was much more variable than AUC and $C_{\max }$. For example, at the $175 \mathrm{mg}$ tid dose, the concentrations varied from 62.6 to $223.1 \mathrm{ng} / \mathrm{mL}$. There was appreciable inter-patient variability, as anticipated for a study with a small number of patients. One of two patients who received $200 \mathrm{mg}$ tid was not included in day 56 analyses because the sample was not available (missed study visit). Per protocol, no patients were receiving 200 mg tid by day 20 (pharmacokinetic sampling was performed on days 6,13 , and 20). Plasma factor D concentration did not change during treatment (Online Supplementary Figure S2A). As anticipated from its mechanism of action, treatment resulted in selective AP inhibition (Figure 1B, upper panel; Online Supplementary Table S4) with no effect on classical pathway activity (Online Supplementary Figure S2B). Notably, AP activity $\leq 10 \%$ was observed at all time points except at 0 and $8 \mathrm{~h}$ when danicopan concentration was lowest (Figure 1B) indicating that the AP activity in patients may not be fully inhibited at the end of the $8 \mathrm{~h}$ dosing period. Pharmacokinetic/pharmacodynamic analysis showed a dose-response relationship between danicopan and AP inhibition (Online Supplementary Figure S2D). 
Table 1. Baseline characteristics and clinical results.

\begin{tabular}{|c|c|c|c|c|c|c|c|c|c|c|}
\hline & $\begin{array}{l}\text { Patient 1* } \\
\text { BL D28 D84 }\end{array}$ & $\begin{array}{c}\text { Patient } 2 \\
\text { BL D28 D84 }\end{array}$ & $\begin{array}{c}\text { Patient } 3^{\S} \\
\text { BL D28 D84 }\end{array}$ & $\begin{array}{l}\text { Patient } 4^{\circ} \\
\text { BL. D28D84 }\end{array}$ & $\begin{array}{l}\text { Patient } 5 \\
\text { BL D28 D84 }\end{array}$ & $\begin{array}{l}\text { Patient } 6 \\
\text { BL. D28 D84 }\end{array}$ & $\begin{array}{l}\text { Patient } 7^{7} \\
\text { BL D28 D84 }\end{array}$ & $\begin{array}{c}\text { Patient } 8 \\
\text { BL. D28 D84 }\end{array}$ & $\begin{array}{l}\text { Patient } 9 \\
\text { BL D28 D84 }\end{array}$ & $\begin{array}{l}\text { Patient 10* } \\
\text { BL D28 D84 }\end{array}$ \\
\hline Danicopan (mg po tid) & $\begin{array}{lll}100 & 150 & 175\end{array}$ & 100150175 & $150175 \quad 200$ & $150175-$ & $\begin{array}{lll}150 & 175 & 175\end{array}$ & 150150150 & $150175-$ & 150175175 & 150175200 & $\begin{array}{lll}150 & 150 & 150\end{array}$ \\
\hline $\mathrm{Hb}(\mathrm{g} / \mathrm{dL})$ & $\begin{array}{lll}7.5 & 9.3 & 8.7\end{array}$ & 11.714 .112 .3 & $\begin{array}{lll}12.0 & 11.8 & 13.7\end{array}$ & $11.712 .6-$ & $\begin{array}{lll}10.4 & 10.2 & 10.9\end{array}$ & 9.211 .211 .4 & $8.710 .2-$ & 9.510 .211 .6 & 10.011 .411 .9 & $\begin{array}{lll}6.9 & 8.4 & 11.1\end{array}$ \\
\hline Reticulocytes $\left(10^{9} / \mu \mathrm{L}\right)$ & $\begin{array}{lll}84 & 76 & 69 \\
\end{array}$ & $\begin{array}{lll}150 & 71 \quad 85 \\
\end{array}$ & $80 \quad 42 \quad 45$ & $170109-$ & $\begin{array}{lll}45 & 55 & 49 \\
\end{array}$ & $\begin{array}{lll}236 & 38 & 105\end{array}$ & $137 \quad 50$ & $217 \quad 74 \quad 48$ & $\begin{array}{lll}171 & 78 & 118\end{array}$ & $249 \quad 109 \quad 130$ \\
\hline Total bilirubin (mg/dL) & 0.530 .700 .41 & 2.340 .880 .47 & $2.40 \quad 0.941 .05$ & $0.580 .35-$ & 0.410 .410 .41 & 1.170 .230 .53 & $0.831 .04-$ & 1.880 .880 .74 & 1.320 .540 .71 & $\begin{array}{lll}1.64 & 0.35 & 0.41\end{array}$ \\
\hline C3d+ RBC (\%) & $0.1-^{\dagger} 0.1$ & $-^{\dagger} \quad 0.1$ & $-^{\dagger} \quad 0.1$ & $0.3--^{\dagger}-$ & $\begin{array}{lll}0.1 & 0.1 & 0.1\end{array}$ & $0.1-^{\dagger} 0.4$ & 0.50 .1 & $0.2-^{\dagger} \quad-^{\mathbb{P}}$ & $0.1-^{\$}$ & $\begin{array}{lll}0.1 & 0.3 & 0.1\end{array}$ \\
\hline FACIT-Fatigue $^{\dagger \dagger}$ & $22 \quad 33 \quad 31$ & $\begin{array}{lll}32 & 47 & 49\end{array}$ & $43 \quad 52$ & $23 \quad 36 \quad-$ & $22 \quad 23 \quad 40$ & $\begin{array}{lll}20 & 52 & 52\end{array}$ & $49 \quad 51 \quad-$ & $\begin{array}{lll}44 & 49 & 50\end{array}$ & $\begin{array}{lll}39 & 46 & 46\end{array}$ & $47 \quad 52$ \\
\hline
\end{tabular}

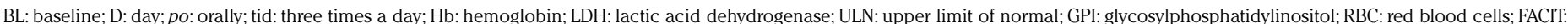

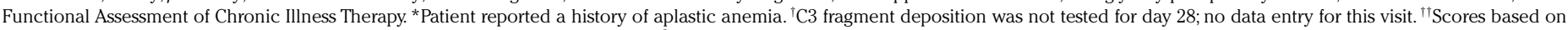

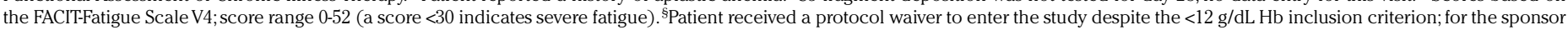

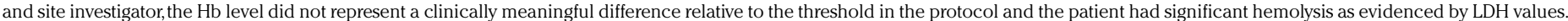

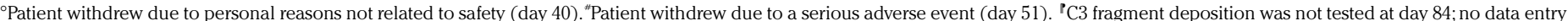
for this visit. ${ }^{\$}$ Incorrect container type.

\section{Efficacy}

\section{Primary endpoint}

Change in $\mathrm{LDH}$ concentration from baseline to day 28 was the primary efficacy endpoint. A significant reduction was observed in all ten patients from a mean value of $5.7 \pm 2.17$ times ULN at baseline to $1.8 \pm 1.03$ times ULN at day $28(P<0.001)$ (Figure 2A, Table 1$)$, demonstrating achievement of the primary endpoint. The percentages of patients showing LDH $<3$ times ULN, $<2$ times ULN, and $<1$ time the ULN were $90 \%, 60 \%$, and $40 \%$, respectively.

\section{Secondary endpoints}

Significant reductions in $\mathrm{LDH}$ from baseline were sustained throughout the study, with values being $2.3 \pm 1.41$ times ULN at day $56(P<0.005)$ and $2.2 \pm 1.04$ times ULN $(P<0.001)$ at day 84 (Figure $2 \mathrm{~A})$. The percentages of patients with $\mathrm{LDH}<3$ times ULN, $<2$ times ULN, and $<1$ time ULN were $71 \%, 43 \%$, and $43 \%$ at day 56 , and $75 \%$, $37.5 \%$, and $25 \%$ at day 84 , respectively. Danicopan is a highly permeable drug and even in patients with high body mass index, plasma concentrations do not appear to cause a clinically significant change in effect. Fluctuations in $\mathrm{LDH}$ indicated that low-level residual intravascular hemolysis remained in most patients, with possible transient exacerbations; this was due to transient weaker AP inhibition around predose periods, as described above, in addition to an increase of susceptible GPI-deficient erythrocytes during treatment (see below). Nevertheless, only two breakthrough hemolytic events were recorded by the investigator as adverse events (Table 2); a third patient experienced recurrent subclinical breakthrough episodes as a consequence of inadequate control of complement activation.

Treatment with danicopan translated into an improvement of anemia: mean hemoglobin increased from 9.8 $\mathrm{g} / \mathrm{dL}$ at baseline (range, 6.9 to $12.0 \mathrm{~g} / \mathrm{dL}$ ) to $10.9 \mathrm{~g} / \mathrm{dL}$ at day 28 (range, 8.4 to $14.1 \mathrm{~g} / \mathrm{dL} ; P<0.005$ ), $10.9 \mathrm{~g} / \mathrm{dL}$ at day 56 (range, 8.5 to $13.1 \mathrm{~g} / \mathrm{dL} ; P<0.005$ ), and $11.5 \mathrm{~g} / \mathrm{dL}$ at day 84 (range, 8.7 to $13.7 \mathrm{~g} / \mathrm{dL} ; P<0.005$ ) (Figure $2 \mathrm{~B}$, Table 1 ). The mean increase from baseline was $0.9 \mathrm{~g} / \mathrm{dL}$ at day 28 and $1.7 \mathrm{~g} / \mathrm{dL}$ at day 84 . In the 12 weeks preceding study entry, two patients received transfusions (Figure $3 A$ ). One of these patients, who had aplastic anemia (not receiving immunosuppressive therapy), had received five transfusions totaling ten units. During treatment, this patient received three transfusions totaling seven units. The sec- ond patient had one transfusion (two units) during breakthrough hemolysis in the setting of a viral infection; in this case, danicopan treatment was not detrimental to the course of the infection, but (irrespective of its possible effect on other complement pathways) did not effectively counteract the transient acute complement activation (possibly due to pharmacokinetic/pharmacodynamic reasons). The remaining patients in the trial were transfusion independent through the 84 days of treatment. Accompanying the control of intravascular hemolysis and improvement of anemia, patient-reported outcomes were assessed. The mean FACIT-Fatigue score at baseline was 34 and increased by 9 and 13 points at days 28 and day 84 , respectively $(P<0.05)$ ( Figure $3 \mathrm{~B}$, Table 1 ).

Control of intravascular hemolysis by danicopan was further confirmed by changes in other laboratory parameters. Danicopan significantly increased the percentage of GPI-deficient erythrocytes $(56 \pm 19.9 \%$ at day 84 vs. $32 \pm 24.6 \%$ at baseline; $P=0.001$ ), whereas no change was observed for GPI-deficient granulocytes (Figure 4A). Total bilirubin decreased after danicopan treatment $(0.6 \pm 0.23$ $\mathrm{mg} / \mathrm{dL}$ at day 84 vs. $1.3 \pm 0.74 \mathrm{mg} / \mathrm{dL}$ at baseline, $P<0.05)$ (Figure 4B). A sustained but not statistically increase in haptoglobin was observed $(15.3 \pm 16.08 \mathrm{mg} / \mathrm{dL}$ at day 84 vs. $\quad 5.8 \pm 2.89 \mathrm{mg} / \mathrm{dL}$ at baseline; $P=0.15$ ) (Online Supplementary Figure $S 3 A$ ), as was a transient decrease in free hemoglobin $(83 \pm 51 \mathrm{mg} / \mathrm{dL}$ at day 28 vs. $138 \pm 132$ $\mathrm{mg} / \mathrm{dL}$ at baseline; $P=0.26$ ) (Online Supplementary Figure $S 3 B)$. Absolute reticulocyte count decreased quickly and was sustained with treatment $\left(81 \pm 33.6 \times 10^{9} / \mathrm{L}\right.$ at day $84 v s$. $154 \pm 69 \times 10^{9} / \mathrm{L}$ at baseline; $P<0.05$ ) (Figure $4 \mathrm{C}$ ). Additional laboratory results can be found in Online Supplementary Table S6.

\section{Exploratory endpoints}

To investigate the mechanistic effect of proximal complement inhibition by danicopan on $\mathrm{PNH}$, complement biomarkers were monitored. Bb fragment, an activation product of factor $\mathrm{B}$, tracks complement AP activation in vivo. Plasma $\mathrm{Bb}$ level was significantly elevated at baseline $(2.24 \pm 0.77 \mu \mathrm{g} / \mathrm{mL})$ compared with that in healthy volunteers $(0.84 \pm 0.212 \mu \mathrm{g} / \mathrm{mL} ; P<0.05)$. After danicopan, the $\mathrm{Bb}$ levels were significantly reduced: $0.84 \pm 0.84 \mu \mathrm{g} / \mathrm{mL}$ at day $28(P<0.005) ; 0.47 \pm 0.09 \mu \mathrm{g} / \mathrm{mL}$ at day $56(P<0.005)$; and $0.47 \pm 0.06 \mu \mathrm{g} / \mathrm{mL}$ at day $84(P<0.005)$ (Figure $1 \mathrm{C})$. In contrast to residual AP activity, $\mathrm{Bb}$ level remained consis- 
tently low irrespective of subtherapeutic plasma danicopan levels in predose periods (Figure $1 \mathrm{~B}$, middle panel). A strong positive correlation was found between $\mathrm{Bb}$ and $\mathrm{LDH}$ (Pearson $\mathrm{r}=0.80, P<0.0001$ ) (Online Supplementary Figure $S 2 E$ ), supporting $\mathrm{Bb}$ as a reliable biomarker of in vivo $\mathrm{AP}$ activation in $\mathrm{PNH}$ and, therefore, its value for monitoring efficacy. Danicopan also showed strong linear correlations with $\mathrm{Bb}$ and $\mathrm{LDH}$ (negative), as did $\mathrm{AP}$ with $\mathrm{Bb}$ and $\mathrm{LDH}$ (positive); there was no correlation of classical pathway activity with any of these parameters (Online Supplementary Table S5), validating the role of danicopan in $\mathrm{AP}$ inhibition and subsequent in vivo changes of $\mathrm{Bb}$ and $\mathrm{LDH}$. Additional laboratory results can be found in Online Supplementary Table S6.

There was a slight increase in serum C3 (114.2 \pm 17.3 $\mathrm{mg} / \mathrm{dL}$ at day 84 vs. $102.2 \pm 20.2 \mathrm{mg} / \mathrm{dL}$ at baseline, $P=0.08)$ (Online Supplementary Figure S2C), likely from reduced C3 consumption because of upstream complement blockade. Importantly, C3 fragment deposition on erythrocytes was very low $(<0.5 \%$ of erythrocytes) throughout treatment (Figure 4A).

sC5b-9 was normal at baseline and remained relatively constant over time (data not shown).

\section{Discussion}

Current PNH treatments target C5 inhibition. ${ }^{10-14}$ Novel complement inhibitors in development aim to address unmet needs of $\mathrm{PNH}$ patients. ${ }^{22}$ Here, we describe a novel approach to $\mathrm{PNH}$ treatment, which aims to change the current paradigm of $\mathrm{PNH}$ therapy by improving hemoglobin levels in addition to reducing hemolysis, with the added advantage and convenience of oral administration. We investigated danicopan, a first-in-class oral factor $D$ inhibitor, which blocks the proximal complement cascade upstream of C5 at the level of AP initiation and amplification. In untreated $\mathrm{PNH}$ patients, danicopan monotherapy resulted in inhibition of intravascular hemolysis, with sig-
A

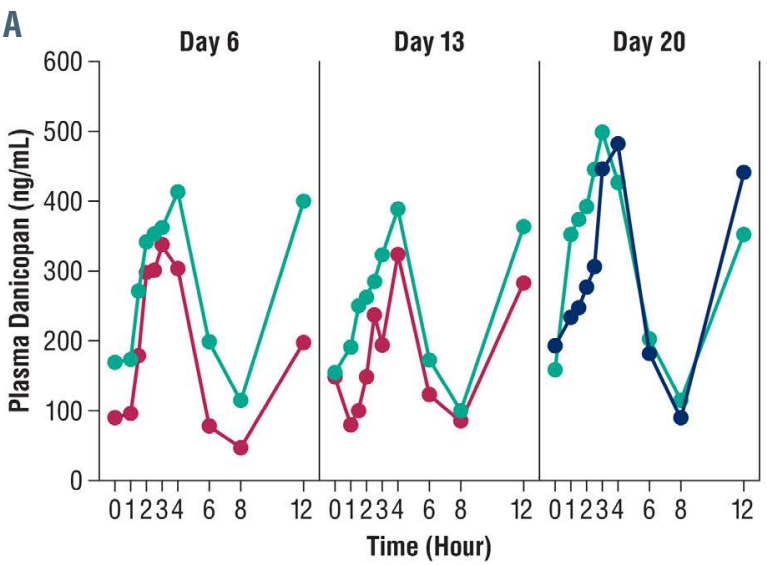

C

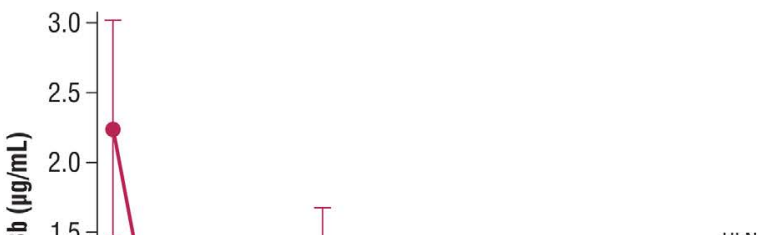

B
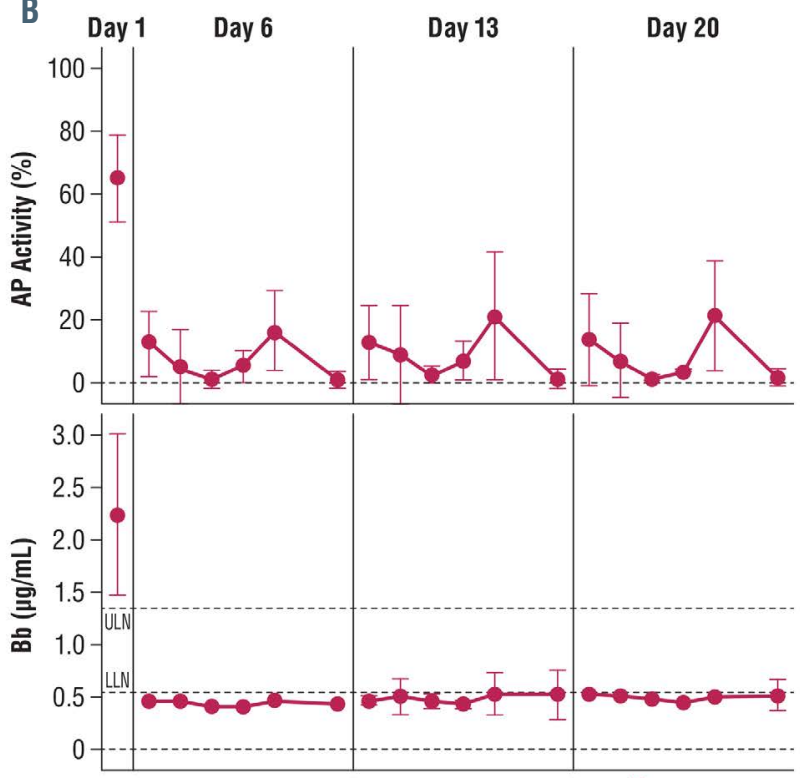

$\lfloor N$

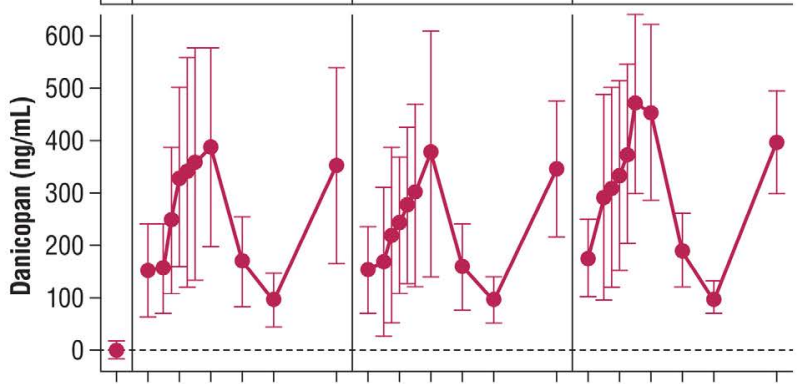

ay 84

6

0.47

$(0.059)^{* * *}$

$0.38,0.54$

Figure 1. Pharmacokinetic-pharmacodynamic evaluation of danicopan. (A) The mean plasma danicopan concentration by dose at hours 0 (predosing), $1,1.5,2$ $2.5,3,4,6,8$ (predosing) and 12 of days 6,13 , and 20. The number of patients is, respectively, two (100 mg tid) and eight (150 mg tid) at day 6 and day 13 , and five (150 mg tid) and five (175 mg tid) at day 20. (B) The mean \pm standard deviation of ex vivo serum alternative pathway (AP) activity, plasma Bb concentration, and plasma danicopan concentration, combining all dosing groups together. Serum AP activity and plasma Bb concentration were determined for a subset of the aforementioned time points by the AP Wieslab assay (Euro Diagnostica) and Bb enzyme-linked immunosorbent assay, respectively. (C) Plasma Bb concentration (mean \pm standard deviation) at baseline (day 1) through the end of the study (day 84) with descriptive statistics. The dashed lines represent the upper and lower limits of normal, which were derived from phase I studies in healthy volunteers (see Assay Methods in the Online Supplement). $\mathrm{N}$ values of $<10$ for plasma Bb on days 42,56 , and 84 reflect the two early discontinuations and additional samples not collected. $* * P<0.005$. tid: thrice daily; ULN: upper limit of normal; LLN: lower limit of normal; SD: standard deviation. 
nificant reductions of $\mathrm{LDH}$ at day 28 (primary endpoint) and throughout treatment duration. In contrast with standard C5 inhibitor therapies, the inhibition of intravascular hemolysis observed during danicopan treatment was not associated with persistently increased bilirubin and reticulocyte count, or with the emergence of C3 deposition on surviving GPI-deficient erythrocytes, in agreement with its anticipated effect on extravascular hemolysis. Concomitant inhibition of intravascular hemolysis and prevention of C3-mediated extravascular hemolysis resulted in improvement of anemia, with a mean hemoglobin gain of $1.7 \mathrm{~g} / \mathrm{dL}$ after 12 weeks of treatment. Consistent with these findings, all patients exhibited significant increases in the percentage of GPI-deficient erythrocytes, confirming the extended half-life of these cells in vivo. Furthermore, all patients had improvements in FACIT-Fatigue quality-of-life measurements; FACITFatigue scores were used in this proof-of-concept study due to the lack of validated instruments for patient-reported outcomes in PNH. Additional patient-reported out-

Table 2. Adverse events.

\begin{tabular}{|c|c|c|c|c|c|c|}
\hline \multirow[b]{2}{*}{ Primary system organ class preferred term* } & \multirow[b]{2}{*}{ N } & \multirow[b]{2}{*}{$\%$ of total $(N=10)$} & \multicolumn{3}{|c|}{ Standard severity grade } & \multirow{2}{*}{ Life-threatening } \\
\hline & & & Milld & Moderate & Severe & \\
\hline Number of subjects reporting & 9 & 90 & 8 & 1 & - & - \\
\hline Number of unique TEAE ${ }^{\dagger}$ & 38 & $\mathrm{NA}$ & & & & \\
\hline Number of subjects with SAE & 1 & 10 & - & - & 1 & - \\
\hline Blood and lymphatic system disorders & 2 & 20 & - & - & - & - \\
\hline Hemolysis & 2 & 20 & 1 & - & 1 & - \\
\hline Gastrointestinal disorders & 3 & 30 & - & - & - & - \\
\hline Abdominal pain & 1 & 10 & - & 1 & - & - \\
\hline Mouth ulceration & 1 & 10 & 1 & - & - & - \\
\hline Nausea & 1 & 10 & 1 & - & - & - \\
\hline Vomiting & 1 & 10 & 1 & - & - & - \\
\hline General disorders and administration site conditions & 4 & 40 & - & - & - & - \\
\hline Fatigue & 1 & 10 & 1 & - & - & - \\
\hline Non-cardiac chest pain & 1 & 10 & 1 & - &.. & - \\
\hline Edema, peripheral & 1 & 10 & 1 & - & - & - \\
\hline Vaccination site erythema & 1 & 10 & 1 & - & - & - \\
\hline Infections and infestations & 5 & 50 & - & - & - & - \\
\hline Pharyngitis & 1 & 10 & - & 1 & - & - \\
\hline Upper respiratory tract infection & 4 & 40 & 3 & 1 & - & - \\
\hline Viral upper respiratory tract infection & 1 & 10 & 1 & - & - & - \\
\hline Injury, poisoning, and procedural complications & 1 & 10 & - & - & - & - \\
\hline Contusion & 1 & 10 & 1 & - & - & - \\
\hline Investigations & 1 & 10 & - & - & - & - \\
\hline Alanine aminotransferase increased & 1 & 10 & - & - & 1 & - \\
\hline Aspartate aminotransferase increased & 1 & 10 & - & - & - & 1 \\
\hline Metabolism and nutrition disorders & 1 & 10 & - & - & - & - \\
\hline Iron deficiency & 1 & 10 & 1 & - & - & - \\
\hline Musculoskeletal and connective tissue disorders & 3 & 30 & - & - & - & - \\
\hline Back pain & 2 & 20 & 2 & - & - & - \\
\hline Myalgia & 1 & 10 & 1 & - & - & - \\
\hline Nervous system disorders & 4 & 40 & - & - & - & - \\
\hline Headache & 4 & 40 & 4 & - & - & - \\
\hline Psychiatric disorders & 1 & 10 & - & - & - & - \\
\hline Irritability & 1 & 10 & 1 & - & - & - \\
\hline Renal and urinary disorders & 3 & 10 & - & - & - & - \\
\hline Hemoglobinuria & 2 & 20 & 2 & - & - & - \\
\hline Paroxysmal nocturnal hemoglobinuria & 1 & 10 & - & 1 & - & - \\
\hline Reproductive system and breast disorders & 1 & 10 & - & - & - & - \\
\hline Dysmenorrhea & 1 & 10 & 1 & - & - & - \\
\hline Skin and subcutaneous tissue disorders & 1 & 10 & - & - & - & - \\
\hline Rash, papular & 1 & 10 & 1 & - & - & - \\
\hline
\end{tabular}

*MedDRA Version $18.1{ }^{\dagger}$ This row represents the number of events; all other rows represent the number of subjects. TEAE: treatment-emergent adverse events; NA: not available; SAE: serious adverse events. 
come instruments will be utilized in a phase III trial. With the caveat of limited sample size and exposure, no safety concerns emerged during the study other than those described; in particular, infectious events were rare, clinically mild, and self-limiting, irrespective of unchanged danicopan treatment. Although the short study duration through the primary endpoint limits the ability to draw robust conclusions, the data do not appear to support the postulated increased risk of infectious complications (due to upstream inhibition of the complement cascade), ${ }^{27}$ in agreement with in vitro data showing that killing of encapsulated and unencapsulated meningococci was nearly unaffected relative to that occurring with eculizumab. ${ }^{28,29}$

Danicopan is the first oral complement inhibitor treat-

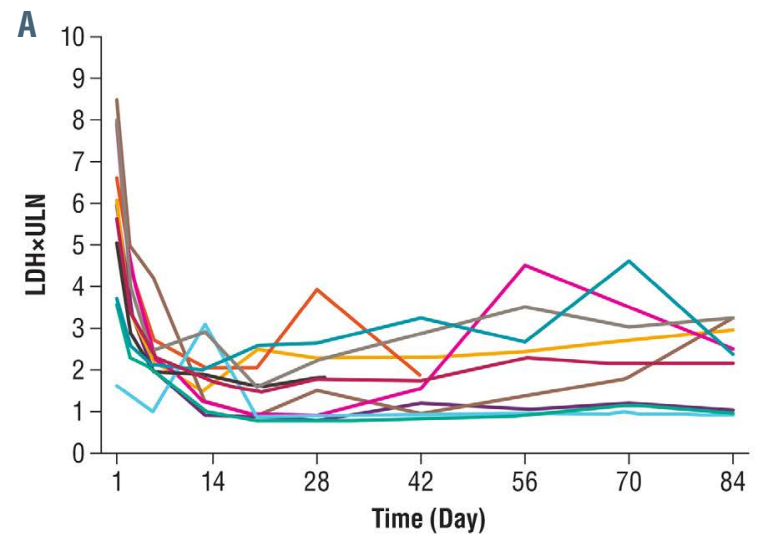

B
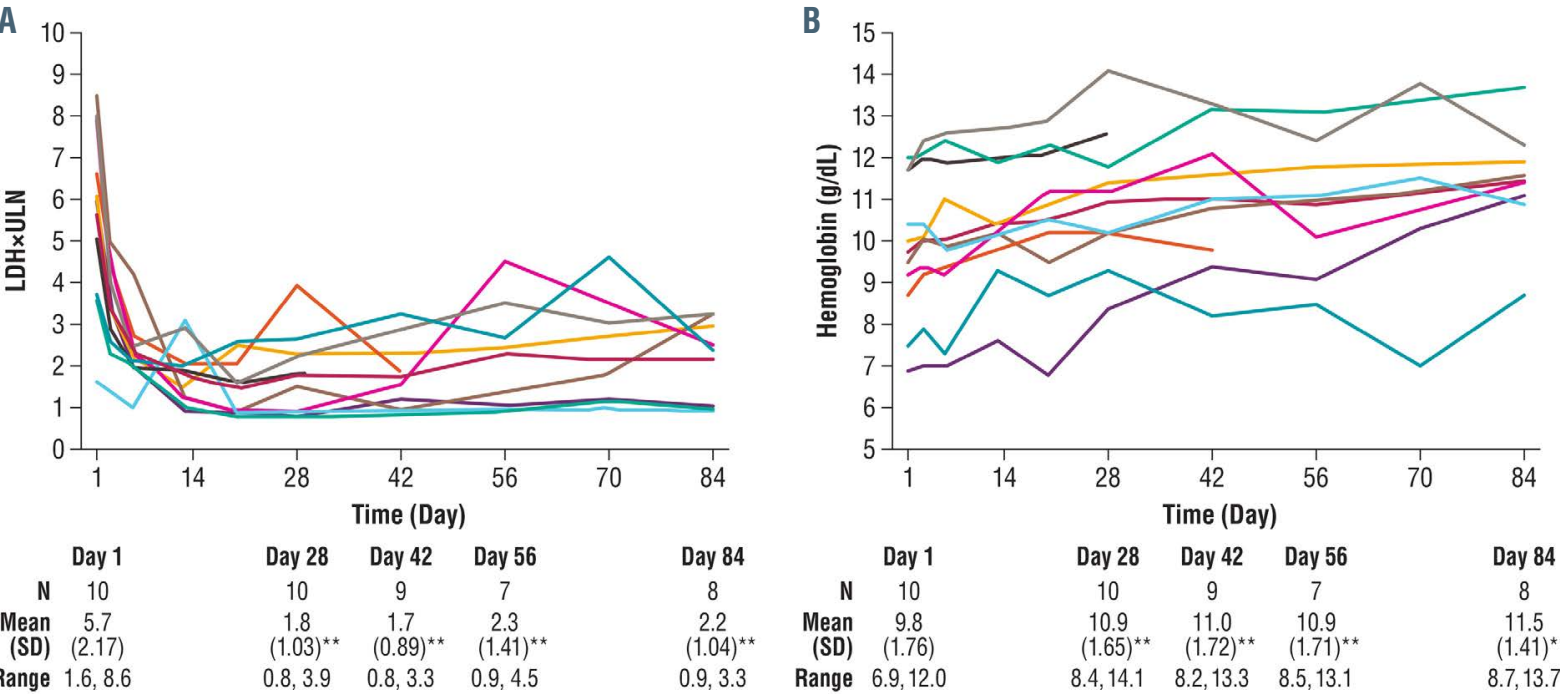

$\begin{array}{cccc}\text { Day 28 } & \text { Day } \mathbf{4 2} & \text { Day } \mathbf{5 6} & \text { Day } \mathbf{8 4} \\ 10 & 9 & 7 & 8 \\ 1.8 & 1.7 & 2.3 & 2.2 \\ (1.03)^{\star \star} & (0.89)^{\star \star} & (1.41)^{\star \star} & (1.04)^{\star \star} \\ 0.8,3.9 & 0.8,3.3 & 0.9,4.5 & 0.9,3.3 \\ & \text { Patient 1 } & \text { - Patient 3 } & \text { - Patient } 5 \\ & \text { - Patient 2 } & \text { Patient 4 } & \text { Patient } 6\end{array}$

$\begin{array}{rc} & \text { Day 1 } \\ \mathbf{N} & 10 \\ \text { Mean } & 9.8 \\ \text { (SD) } & (1.76) \\ \text { Range } & 6.9,12.0\end{array}$

$\begin{array}{ccc}\text { Day } 28 & \text { Day } \mathbf{4 2} & \text { Day } 56 \\ 10 & 9 & 7 \\ 10.9 & 11.0 & 10.9 \\ (1.65)^{\star \star} & (1.72)^{\star \star} & (1.71)^{\star \star} \\ 8.4,14.1 & 8.2,13.3 & 8.5,13.1\end{array}$

Day 84

7

(SD) (2.17)

- Patient 2 -Patient 4 - Patient 6

5 - Patient 7 - Patient 9 - Mean

- Patient 8 - Patient 10

Figure 2. Effect of danicopan on lactate dehydrogenase and hemoglobin levels. (A) Change in lactate dehydrogenase (LDH) concentration from baseline (day 1) to day 28 was the primary efficacy endpoint. LDH reduction per patient is shown here including the reduction from a mean value of $5.7 \pm 2.17$ times upper limit of normal (ULN) at baseline to $1.8 \pm 1.03$ times ULN at day $28(P<0.001)$ demonstrating achievement of the primary endpoint. A significant mean LDH reduction from baseline was sustained throughout the study up to day $84(2.2 \pm 1.04$ times ULN; $P<0.001)$. (B) Per patient effects on hemoglobin with a mean group increase from $9.8 \mathrm{~g} / \mathrm{dL}$ at baseline (day 1) (range, 6.9 to $12.0 \mathrm{~g} / \mathrm{dL}$ ) to $10.9 \mathrm{~g} / \mathrm{dL}$ at day 28 (range, 8.4 to $14.1 \mathrm{~g} / \mathrm{dL} ; P<0.005$ ), and $11.5 \mathrm{~g} / \mathrm{dL}$ at day 84 (range, 8.7 to $13.7 \mathrm{~g} / \mathrm{dL}, P<0.005$ ). Note, patient 3 received a protocol waiver to enter the study despite the $<12 \mathrm{~g} / \mathrm{dL}$ hemoglobin inclusion criterion. For the sponsor and site investigator, the hemoglobin level did not represent a clinically meaningful difference relative to the threshold in the protocol and the patient had significant hemolysis, as evidenced by LDH values. $* * P<0.005$; SD:standard deviation.
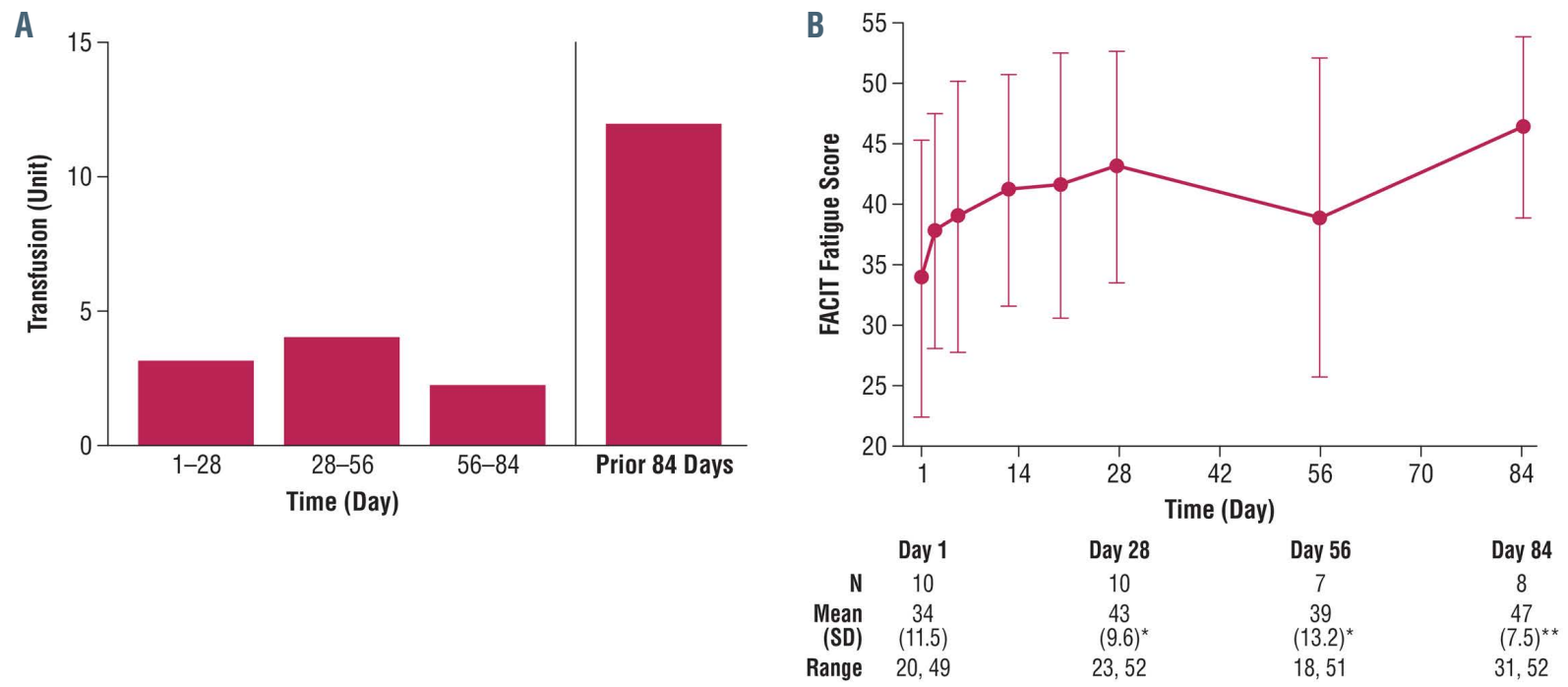

Figure 3. Effect of danicopan on blood transfusions and Functional Assessment of Chronic Illness Therapy-Fatigue score. (A) Two patients required transfusions during the trial, for a total of nine units on four occasions over 84 days. The transfusion history ( 84 days prior to screening through to the end of the study; sum for all patients) is provided. (B) Mean Functional Assessment of Chronic Illness Therapy-Fatigue score values ( \pm standard deviation) at baseline (day 1 ) through to the end of the study (day 84) with descriptive statistics. The range of scores was 0 to 52 ; a score of $<30$ indicates severe fatigue. $* P<0.05$; $* * P<0.005$. Note, data were not obtained for one patient at day 56. FACIT: Functional Assessment of Chronic Illness Therapy; SD: standard deviation. 
ment demonstrating efficacy and safety in $\mathrm{PNH}$ patients as monotherapy in a phase II study. The clinical effects observed were achieved irrespective of low-level, residual intravascular hemolysis, which remained detectable in some patients. This residual intravascular hemolysis is likely the consequence of the increase of GPI-deficient erythrocytes susceptible to complement-mediated hemolysis and the pharmacokinetic/pharmacodynamic characteristics of danicopan. Danicopan plasma level and AP activity also correlated with in vivo biomarkers (LDH and $\mathrm{Bb}$ ), and $\mathrm{Bb}$ seems a reliable indicator of complement activation in vivo during danicopan treatment. Based on the results of the phase I single-dose and multiple ascendingdose trials in healthy human volunteers, $200 \mathrm{mg}$ tid was shown to be both safe and efficacious. ${ }^{26}$ However, in this cohort of patients, full blockade of AP activity was not consistently achieved in all patients, irrespective of individual dose adjustment and the broad dose ranges used during the study. These observations suggest that residual intravascular hemolysis is due to low residual AP activity observed in some patients, which may be better inhibited by a more potent second-generation factor $\mathrm{D}$ inhibitor analog that will be assessed for safety and efficacy in a phase II trial (Clinicaltrials.gov, NCT04170023). In a concurrent phase II trial, patients $(n=12)$ on a stable regimen of eculizumab with hemoglobin $<10 \mathrm{~g} / \mathrm{dL}$ and who were transfusion dependent $(\geq 1$ red blood cell transfusion within 12 weeks of screening) received oral danicopan 100-150 mg tid, with possible response-based dose escalation to $200 \mathrm{mg}$ tid at predefined time points. The addition of danicopan led to clinically and statistically significant reductions in frequency of red blood cell transfusions and in the number of transfused units in patients compared to those in patients with a history of eculizumab treatment alone. ${ }^{30}$

Clinical development of proximal complement inhibitors has been motivated by the description of C3mediated extravascular hemolysis as a mechanism driving significant anemia and limiting hematologic benefit in some $\mathrm{PNH}$ patients on eculizumab and other C5 inhibitors. ${ }^{17,31}$ Indeed, it is conceivable that, in combination with C5 inhibitors, proximal inhibitors can address C3-mediated extravascular hemolysis by preventing the generation of C3 convertase, eventually leading to better hematologic response in $\mathrm{PNH}$ patients. ${ }^{17,31}$ Moreover, it has been hypothesized that proximal inhibitors, by preventing generation of downstream C5 convertases, could be effective even in the absence of terminal inhibitors (i.e., eculizumab or other C5 inhibitors). Danicopan is the first compound demonstrating that proximal complement inhibitors can be used safely and effectively as monother-
A

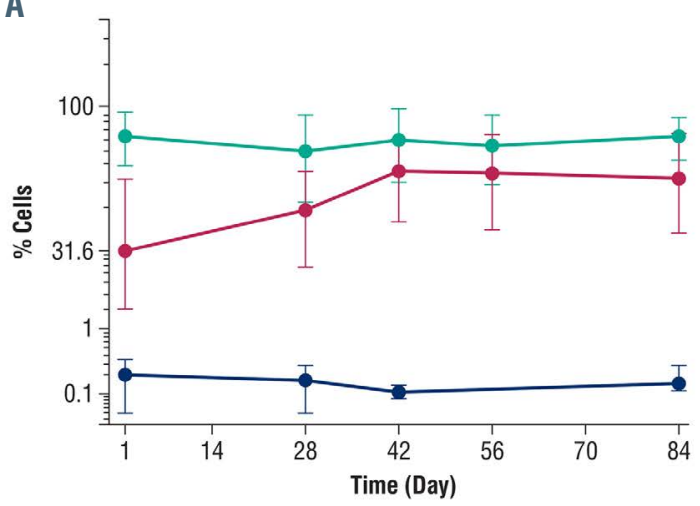

B

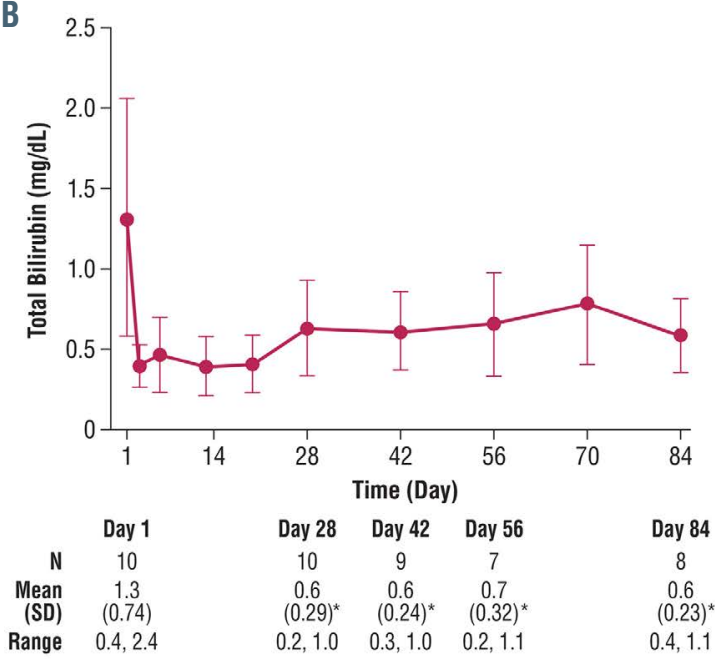

$\rightarrow$ GPI-deficient granulocytes $\rightarrow$-GPI-deficient erythrocytes $\rightarrow$ C3d positive erythrocytes

C

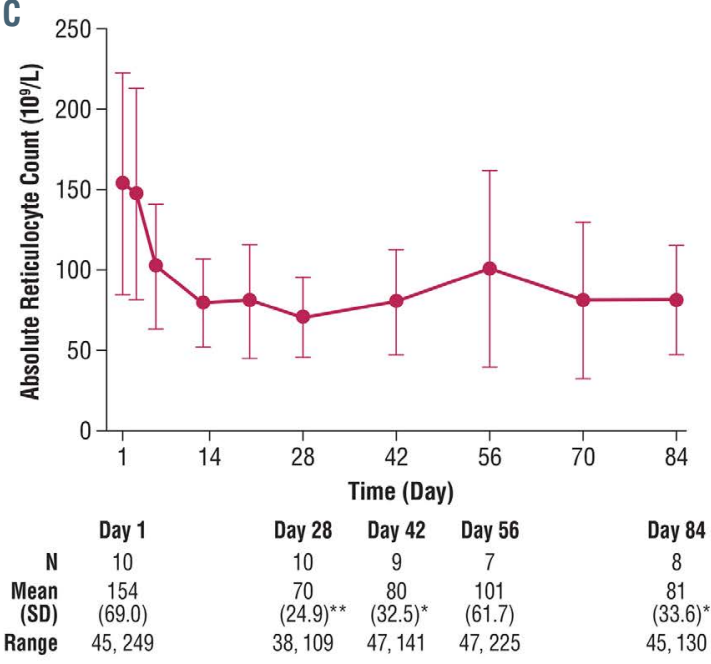

Figure 4. Additional clinical efficacy evaluation of danicopan. (A) The mean ( \pm standard deviation) clone size percentages displayed for GPI-deficient erythrocytes and granulocytes at baseline (day 1) through to the end of the study (day 84 ) and mean ( \pm standard deviation) percentage of erythrocytes with C3 fragment deposition with descriptive statistics for GPI-deficient erythrocytes. Descriptive statistics for GPI-deficient granulocytes and erythrocytes with C3 fragment deposition are listed in Online Supplementary Table S6. Erythrocytes with C3 fragment deposition are defined as erythrocytes that stain positive for anti-C3d antibody. (B) Total bilirubin at baseline (day 1) through to the end of the study (day 84) with descriptive statistics. Data for one patient were not obtained at day 56. (C) Absolute reticulocyte count at baseline (day 1 ) through to the end of the study (day 84 ) with descriptive statistics. Data for one patient were not obtained at day 56. $* P<0.05$; $* * P<0.005$. SD: standard deviation; GPI: glycosylphosphatidylinositol. 
apy in PNH. By disabling the initiating event of complement activation, danicopan prevents generation of $\mathrm{C} 5$ convertases, obviating the need for downstream C5 inhibition. Additionally, specific targeting of AP can preserve classical and lectin pathway-mediated antimicrobial activity. The landscape of complement inhibition in the treatment of PNH will continue to evolve with the availability of proximal inhibitors. In addition to danicopan, other oral agents targeting the $\mathrm{AP}$, such as factor $\mathrm{B}$ inhibitors, are under investigation for the treatment of $\mathrm{PNH}^{22,31,32}$ Proximal complement inhibitors also include subcutaneously administered agents targeting C3; one of these agents was reported to be effective in a recent phase III trial in patients with $\mathrm{PNH}^{33}$ All of these approaches look promising for the treatment of $\mathrm{PNH}$ and clinical data should tell us very soon what are the viable treatment options in terms of safety and efficacy, and how we can best utilize them in the appropriate patients (i.e., monotherapy vs. add-on treatment). Preclinical data seem to suggest that $\mathrm{AP}$ inhibitors may be as effective as terminal complement blockade with clinical differences mostly due to the specific pharmacokinetic/pharmacodynamic profile of the individual inhibitor, rather than to its target in the complement cascade. ${ }^{22,31}$ Indeed targeting the AP, as $\mathrm{PNH}$ is a disease due to AP dysregulation (i.e., continuous, spontaneous C3 tick-over, eventually exacerbated at times of additional complement activation), possible applications in other diseases will require an understanding of their pathogenic mechanisms. ${ }^{34}$

In conclusion, in this study danicopan appeared to be well-tolerated and showed clinically meaningful inhibition of intravascular hemolysis and hemoglobin improvement in untreated PNH patients. This is the first evidence that a proximal complement inhibitor, used as monotherapy, can have a clinical impact on $\mathrm{PNH}$ by inhibiting intravascular hemolysis and preventing extravascular hemolysis. While second-generation compounds with improved pharmacokinetics and pharmacodynamics are in development, this study paves the way to improved hematologic response and novel standards of care, with an easier mode of administration, for hemolytic $\mathrm{PNH}$ patients.

\section{Disclosures}

$A M R$ has received research support from Alexion, Novartis, Alnylam and Ra Pharma, lecture fees from Alexion, Novartis, Pfizer and Apellis, served as a member of advisory/investigator boards for Achillion, Alexion, Apellis, Biocryst, Novartis, Roche, and Samsung, and served as a consultant for Amyndas. AGK has served on advisory boards for Alexion, Celgene, Novartis, Ra Pharma, and Regeneron and has received travel grants from Achillion, Celgene, and Ra Pharma. JWL has received grants from Alexion and Achillion, has served as a member of advisory boards for Alexion and Apellis, has received honoraria from Alexion, and has served as a consultant for AlloVir. JPM has received lecture honoraria from Alexion, Novartis, and Celgene and served as a consultant for Novartis. $R N$ has received lecture fees from Alexion. RB has served on scientific advisory boards for Achillion and Alexion. PB has served as a member of advisory boards for Merck, Janssen, Roche and AbbVie, has served as an associate editor for the Internal Medicine Journal, and received research funding from Roche, Beigene, and Amgen. MH is an employee of Alexion and has equity ownership in the same company. MG was an employee of Achillion, Inc., a subsidiary of Alexion Pharmaceuticals, Inc. and had equity ownership in the same company.

\section{Contributions}

$M H$ and $M G$ developed the study protocol, with the contribution of $A M R, A G K, R N, R B$ and $P B ; A M R, A G K, J W L$, and $P B$ recruited and treated patients, and collected the data; $A M R, M H$ and $M G$ analyzed and interpreted the data, and wrote the manuscript; $A G K, J W L, J P M, R N, R B$ and $P B$ contributed to the manuscript and approved its final version.

\section{Acknowledgments}

We thank the patients and investigators, as well as their staff, who participated in this trial: Serena Marotta, Luana Marano and Fabiana Cacace (Naples), Petra Muus and Shreyans Gandhi (London), Federica Barone (Florence), Sung-Soo Park (Seoul), and Paul Hamilton (Auckland).

\section{Funding}

The study was sponsored and entirely supported by Achillion Inc., a subsidiary of Alexion Pharmaceuticals, Inc. We thank Heather Robison (an employee of Achillion Inc., a subsidiary of Alexion Pharmaceuticals, Inc.) and Steven Podos, Danny Shin and Julia Catini (Alexion employees and former employees of Achillion Inc., a subsidiary of Alexion Pharmaceuticals, Inc.) for their assistance in writing this manuscript and The Curry Rockefeller Group (funded by Achillion Inc., a subsidiary of Alexion Pharmaceuticals, Inc.) for their editorial assistance.

\section{Data sharing}

Alexion will consider requests for disclosure of clinical study participant-level data provided that participant privacy is assured through methods such as data de-identification, pseudonymization, or anonymization (as required by applicable law), and if such disclosure was included in the relevant study informed consent form or similar documentation. Qualified academic investigators may request participant-level clinical data and supporting documents (statistical analysis plan and protocol) pertaining to Alexion-sponsored studies. Further details regarding data availability and instructions for requesting information are available in the Alexion Clinical Trials Disclosure and Transparency Policy at http://alexion.com/research-development.

\section{References}

1. Luzzatto L, Notaro R. Paroxysmal nocturnal hemoglobinuria. In: Handin RI, Lux SE, Stossel TP, eds. Blood, Principles and Practice of Hematology. 2nd edition. Philadelphia: Lippincott Williams \& Wilkins; 2003:319-334.

2. Takeda J, Miyata T, Kawagoe K, et al. Deficiency of the GPI anchor caused by a somatic mutation of the PIG-A gene in paroxysmal nocturnal hemoglobinuria.
Cell. 1993;73(4):703-711.

3. Miyata T, Yamada N, Iida Y, et al. Abnormalities of PIG-A transcripts in granulocytes from patients with paroxysmal nocturnal hemoglobinuria. N Engl J Med. 1994;330(4):249-255

4. Medof ME, Gottlieb A, Kinoshita T, et al. Relationship between decay accelerating factor deficiency, diminished acetylcholinesterase activity, and defective terminal complement pathway restriction in paroxysmal nocturnal hemoglobinuria ery- throcytes. J Clin Invest. 1987;80(1):165-174

5. Rotoli B, Luzzatto L. Paroxysmal nocturnal haemoglobinuria. Baillieres Clin Haematol. 1989;2(1):113-138.

6. Luzzatto L, Risitano AM. Advances in understanding the pathogenesis of acquired aplastic anaemia. Br J Haematol. 2018; 182(6):758-776

7. Nicholson-Weller A, March JP, Rosenfeld SI, Austen KF. Affected erythrocytes of patients with paroxysmal nocturnal hemoglobinuria are deficient in the complement 
